\title{
Cogan-Reese Syndrome
}

National Cancer Institute

\section{Source}

National Cancer Institute. Cogan-Reese Syndrome. NCI Thesaurus. Code C84644.

A very rare syndrome characterized by eye abnormalities that lead to vision impairment.

These abnormalities include iris atrophy, decreased corneal endothelium, iris nodules, adhesion of the iris to the lens and glaucoma. 